\title{
Tendência da situação epidemiológica e operacional da hanseníase no estado de Minas Gerais (2008 - 2018)
}

Trend of the epidemiological and operational situation of leprosy in the state of Minas Gerais (2008 - 2018)

Tendencia de la situación epidemiológica y operacional de la lepra en el estado de Minas Gerais (2008 - 2018)

\begin{abstract}
RESUMO
Objetivo: Analisar a tendência da situação epidemiológica e operacional da hanseníase no estado de Minas Gerais, período 2008 a 2018. Método: Estudo ecológico de séries temporais. Foram utilizados os indicadores taxa de detecção anual de casos novos de hanseníase e proporção de contatos examinados de casos novos de hanseníase. Os dados foram extraídos do departamento de informática do Sistema Único de Saúde do Brasil, disponibilizadas pelo Ministério da Saúde por meio do acesso a sua página na internet. Utilizou-se análise linear generalizada de Prais-Winsten para análise de tendência. Resultados: Foram registrados 14.991 casos de hanseníase no período. 0 estado de Minas Gerais apresentou tendência decrescente $(-6,74)$ para detecção geral e tendência crescente $(3,38)$ para a proporção de contatos examinados. Conclusão: Apesar do comportamento favorável dos indicadores analisados, 0 estado ainda mantém média endemicidade para a doença e uma regular capacidade dos serviços em realizar a vigilância de contatos. DESCRITORES: Doenças Negligenciadas; Estudos de Séries Temporais; Hanseníase; Medidas em Epidemiologia.
\end{abstract}

\section{ABSTRACT}

Objective: To analyze the trend of the epidemiological and operational situation of leprosy in the state of Minas Gerais, from 2008 to 2018. Methods: Ecological study of time series. The annual rate of detection of new leprosy cases and proportion of examined contacts of new leprosy cases were used. The data were extracted from the informatics department of the Brazilian Unified Health System, made available by the Ministry of Health through access to its website. Prais-Winsten generalized linear analysis was used for trend analysis. Results: There were 14.991 leprosy cases in the period. The state of Minas Gerais showed a decreasing trend (-6.74) for general detection and an increasing trend (3.38) for the proportion of contacts examined. Conclusion: Despite the favorable behavior of the analyzed indicators, the state still maintains average endemicity for the disease and a regular capacity of the services to carry out contact surveillance.

DESCRIPTORS: Neglected Diseases; Time Series Studies; Hansen's disease; Measures in Epidemiology.

\section{RESUMEN}

Objetivo: Analizar la tendencia de la situación epidemiológica y operativa de la lepra en el estado de Minas Gerais, de 2008 a 2018. Métodos: Estudio ecológico de series de tiempo. Se utilizó la tasa anual de detección de nuevos casos de lepra y la proporción de contactos examinados de nuevos casos de lepra. Los datos fueron extraídos del departamento de informática del Sistema Único de Salud de Brasil, puesto a disposición por el Ministerio de Salud a través del acceso a su sitio web. Para el análisis de tendencias se utilizó el análisis lineal generalizado de Prais-Winsten. Resultados: Hubo 14.991 casos de lepra en el período. El estado de Minas Gerais mostró una tendencia decreciente $(-6,74)$ para la detección general y una tendencia creciente $(3,38)$ para la proporción de contactos examinados. Conclusión: A pesar del comportamiento favorable de los indicadores analizados, el estado aún mantiene una endemicidad promedio de la enfermedad y una capacidad regular de los servicios para realizar vigilancia de contactos.

DESCRIPTORES: Enfermedades desatendidas; Estudios de series temporales; Enfermedad de Hansen; Medidas en Epidemiología.

RECEBIDO EM: 14/08/2020 APROVADO EM: 24/08/2020

\section{Marla Ariana Silva}

Graduanda em Enfermagem pela Universidade do Estado de Minas Gerais (UEMG), Unidade Divinópolis, Brasil. ORCID: 0000-0003-0136-7122

\section{Karen dos Santos Lago}

Graduanda em Enfermagem pela Universidade do Estado de Minas Gerais (UEMG), Unidade Divinópolis, Brasil. ORCID: 0000-0002-4502-5098 


\section{Cláudia Martins da Costa}

Graduanda em Enfermagem pela Universidade do Estado de Minas Gerais (UEMG), Unidade Divinópolis, Brasil. ORCID: 0000-0001-5904-6829

\section{Isabela de Caux Bueno}

Enfermeira. Doutoranda do Programa de Pós-Graduação em Enfermagem da Universidade Federal de Minas Gerais (UFMG), Brasil.

ORCID: 0000-0003-4501-5989

\section{Rayssa Nogueira Rodrigues}

Enfermeira. Doutora em enfermagem pela Universidade Federal de Minas Gerais (UFMG), Brasil. Docente do curso de Enfermagem da Universidade do Estado de Minas Gerais (UEMG), Unidade Divinópolis.

ORCID: 0000-0002-4772-4968

\section{INTRODUÇÃO}

A hanseníase é uma doença infectocontagiosa crônica, cujo agente etiológico é o Mycobacterium leprae (M. leprae). Esse bacilo tem a capacidade de infectar grande número de indivíduos (alta infectividade), no entanto poucos adoecem (baixa patogenicidade). A doença pode acometer pessoas de ambos os sexos e qualquer idade. Entretanto, a sua progressão é lenta e o período de incubação é prolongado, podendo durar anos ${ }^{(1)}$.

Mesmo sendo conhecida como uma das doenças mais antigas da humanidade, ainda constitui um importante problema de saúde pública. Segundo dados da Organização Mundial da Saúde (OMS), o Brasil é o segundo colocado em número absoluto de casos novos registrados no mundo e o líder das Américas ${ }^{(2)}$. Dentro do país, o quadro é ainda mais diversificado.

Particularmente em relação ao estado de Minas Gerais, em 2017, foram notificados 990 novos casos, o que corresponde a uma taxa de detecção de 4,71 casos por $100 \mathrm{mil}$ habitantes ${ }^{(3)}$. Embora esse valor classifique o estado em média endemicidade e apresente indícios da manutenção da endemia ${ }^{(4)}$, estudo recente não identificou clusters de risco em Minas Gerais ${ }^{(5)}$. Esse fato coloca em debate se a situação epidemiológica no estado refere a baixa transmissibilidade ou a baixa detecção pelos profissionais de saúde, uma vez que Minas Gerais é uma área historicamente endêmica para a doença.

Muitos são os fatores envolvidos em sua distribuição e propagação. Além dos determinantes genéticos ${ }^{(6)}$ e imunológicos ${ }^{(7)}$, a hanseníase é influenciada pelas condições socioeconômicas e por questóes operacionais dos serviços de saúde ${ }^{(8)}$. Ainda, a falta de informação gera muito preconceito, fato que prejudica a sua identificação e facilita o contágio de mais pessoas ${ }^{(9)}$.

A principal estratégia brasileira para alcançar baixos níveis endêmicos baseia-se na organização de uma rede de atenção com a integração das ações de controle, que inclui dentre outras, a vigilância de contatos ${ }^{(10)}$. Os contatos representam uma população com maior risco de adoecimento do que a população em geral devido à maior probabilidade de exposição ao bacilo ${ }^{(11)}$. Inclusive, para efetuar o monitoramento e avaliação da eficácia e efetividade das medidas de vigilância realizadas pelos serviços de saúde, o Ministério da Saúde orienta a construção e análise do indicador "proporção de contatos examinados de casos novos de hanseníase diagnosticados nos anos das coorte" ${ }^{\prime(12)}$.

Para, no entanto, avaliar o comportamento dos indicadores, faz-se necessário analisar um período de tempo relativamente longo, pois a vigilância epidemiológica da doença pode variar operacionalmente de ano para ano, mas não de forma estável, por uma déca$\mathrm{da}^{(13)}$. E dentre as estratégias de investigação utilizada na epidemiologia e saúde pública, tem-se a análise da distribuição temporal de eventos do processo saúde-doença, também denominados análise de tendência. Este tipo de estudo contribui para a compreensão das variaçôes temporais de determinantes das condições de saúde, de fatores de risco ou proteção e para a formulação de hipóteses sobre a causalidade de várias doenças ${ }^{(14)}$.

Assim, considerando que a hanseníase constitui um problema de saúde púbica no
Brasil e que a ausência de clusters em Minas Gerais pode estar indicando uma fragilidade do serviço, este estudo visa analisar a tendência da taxa de detecção de casos novos e a proporção de contatos examinados de casos de hanseníase no estado de Minas Gerais, período 2008 a 2018.

Pretende-se com este estudo nortear políticas públicas e orientar a reorganização dos serviços de saúde para fortalecer sua atuação no controle dessa doença. Além disso, contribuir para maior divulgação de estudos sobre uma doença que é tão negligenciada.

\section{MÉTODO}

Trata-se de um estudo ecológico de séries temporais que foi realizado no estado de Minas Gerais. Composto por 853 municípios, o estado possui uma população total estimada de 20.997.560 habitantes, sendo o segundo maior em número de habitantes do Brasil ${ }^{(15)}$.

As informações dos casos de hanseníase, do período 2008 a 2018, foram retiradas do departamento de informática do Sistema Único de Saúde (SUS) do Brasil (DATASUS), disponibilizadas pelo Ministério da Saúde por meio do acesso a sua página na internet. $\mathrm{O}$ período de intervalo de tempo relativamente longo foi utilizado, visto que variações operacionais, que porventura tenham ocorrido, estarão diluídas neste período, proporcionando condições para uma melhor aproximação da realidade da endemia.

Utilizou-se para o tratamento dos dados o software Microsoft Office Excel (2016) que contemplou a estruturação de um banco de dados com os seguintes indicadores: 1- Taxa de detecção anual de casos novos de hanseníase 
por 100 mil habitantes , 2- Proporção de contatos examinados de casos novos de hanseníase diagnosticados nos anos das coorte ${ }^{(16)}$.

Para a análise de tendência foi utilizado o software Stata (versão 12), e empregado o modelo de regressão linerar de Prais-Winsten, em que a variável independente $(\mathrm{x})$ foi o ano (2008 a 2018) e a variável dependente (y) os indicadores supracitados. Esse modelo é indicado para corrigir a autocorrelação serial em séries temporais ${ }^{(17)}$.

Inicialmente, foi realizada a transformação logarítmica dos valores de y para reduzir a heterogeneidade da variância dos resíduos da análise de regressão ${ }^{(17)}$. Posteriormente, foi realizada a aplicação do modelo de Prais-Winsten.

Para identificação da variação percentual média anual (Annual Percent Change - APC), os valores do coeficiente b1 correspondentes a cada um dos indicadores foram aplicados à seguinte fórmula: $\mathrm{APC}=-1+10$ [b1] ${ }^{*} 100 \%$. Por fim, foram calculados os

\section{Figura 1 - Taxa de detecção de casos novos e proporção de contatos} examinados de casos novos de hanseníase (2008 a 2018)

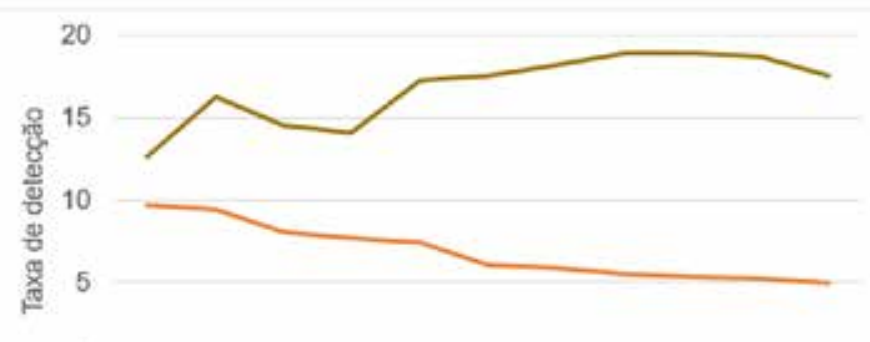

20

0

20082009201020112012201320142015201620172018

- Taxa de deteçăo de casos novos de hanseniase

-Proporçáo de contalos examinados de casos novos de hanseniase

Tabela 1 - Tendência e variação percentual anual das taxas de detecção de casos novos e proporção de contatos examinados de casos novos de Hanseníase (2008 a 2018)

Indicadores

(\%)

IC $95 \%$

p

$-6,74$

$(-8,15 ;-5,30)$

$<0,001$

Decrescente

novos

Proporção de contatos examinados de casos novos

3,38

$(1,73 ; 5,05)$

0,001

Crescente

Tendência

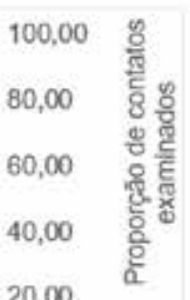

0,00

intervalos de confiança (IC) 95\% das medidas de variação, mediante a aplicação das sepositiva; decrescente, quando a taxa de variaço foi signicativamente negativa; e estacio, quando aceita-se a hipótese nula que 作

$\mathrm{O}$ estudo atendeu às determinações da Resolução 466/12 do Conselho Nacional de Saúde ${ }^{(18)}$.

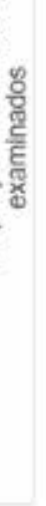

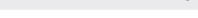

\section{RESULTADOS}

No período de estudo foram notificados 14.991 casos de hanseníase. A taxa de detecção de casos novos no estado de Minas Gerais foi de 6,82 por 100 mil habitantes e a proporção de contatos examinados de casos novos foi 83,76\%. Observa-se na Figura 1 que a taxa de detecção na população geral e a proporção de contatos examinados apresentaram diminuição e aumento dos valores no período em estudo, respectivamente. Destaca-se, entretanto, uma queda na proporção de contatos examinados entre os anos de 2009 e 2011.

$\mathrm{Na}$ tabela 1 é possível observar que houve uma tendência decrescente para a taxa de detecção de casos novos e tendência crescente para a proporção de contatos examinados.

\section{DISCUSSÃO}

A partir dos resultados obtidos, observou-se que houve um decréscimo na taxa de detecção de novos casos de hanseníase no estado durante o intervalo analisado, o que demonstra que Minas Gerais, nesse período, acompanhou a tendência nacional ${ }^{(19)}$. No entanto, Minas Gerais permanece com uma área de média endemicidade para a doença(10). Além disso, quando as taxas são parte dos esforços das equipes, o número de pacientes recém-detectados aumenta substancialmente, no entanto, nos anos subsequentes, os números tendem a diminuir ${ }^{(20)}$. Embora, essa afirmativa seja plausível, não se pode descartar a possibilidade desse estudo não ter captado esse aumento. $\mathrm{O}$ processo de descentralização das ações de controle da hanseníase para a Atenção Primária à Saúde (APS) ganhou destaque após o ano 2000, atingindo um pico em $2003^{(21)}$.

É fato que a mudança do programa vertical de atenção à hanseníase para um serviço descentralizado de controle da doença colheu resultados satisfatórios ${ }^{(22)}$. A descentralização permite uma maior aproximação do serviço com o usuário do Sistema Único de Saúde (SUS), impactando positivamente na acessibilidade ao tratamento, para a prevenção de incapacidades e para a redução da exclusão social. Além disso, os profissionais desse ponto de atenção desenvolvem tra- 


\section{artigo}

Ariana Silva, M.; Lago, K.S.; Costa, C.M.; Bueno, I.C.; Rodrigues, R.N.;

Tendência da situação epidemiológica e operacional da hanseníase no estado de Minas Gerais (2008 - 2018)

balhos individuais, com a família e com a comunidade, reduzindo o estigma e o desconhecimento sobre uma doença curável e com tratamento gratuito ${ }^{(23)}$. Um estudo realizado na Etiópia, as ações de controle da hanseníase foram descentralizadas para serviços básicos de saúde, com o intuito de aproximar a população do diagnóstico precoce, tratamento oportuno e acompanhamento dos casos, resultados positivos já foram evidenciados ${ }^{(24)}$. Outro estudo em Orissa, na Índia, mostrou que a maior cobertura da APS contribuiu para a melhoria dos indicadores epidemiológicos da hanseníase ${ }^{(25)}$.

Embora a cobertura da APS em Minas Gerais seja de $86,63 \%$, permanece fragilidades no controle da doença. A tendência crescente do indicador proporção de contatos examinados poderia apontar a efetividade do serviço, no entanto, a média encontrada para o período analisado classifica o estado como regular. Segundo o Ministério da Saúde, o percentual de contatos examinados para ser classificado como bom teria de apresentar um percentual superior a $90 \%{ }^{(10)}$.

Dentre as ações realizadas pelos profissionais para a vigilância de controle dos contatos, menciona-se o exame dermatoneurológico, a fim de identificar precocemente sinais da doençą ${ }^{(26)}$. O Ministério da Saúde orienta avaliar anualmente, durante cinco anos, todos os contatos não doentes, quer sejam familiares ou sociais. Após esse período os contatos devem ser liberados da vigilância, devendo, entretanto, serem esclarecidos quanto à possibilidade de aparecimento de sinais e sintomas sugestivos da hanseníase no futuro.

Além do exame dermatoneurológico, o Ministério da Saúde recomendada a imunoprofilaxia dos contatos com a vacina $\mathrm{Ba}$ cillus Calmette-Guérin (BCG), no entanto, pouco se sabe sobre sua implementação( ${ }^{(27)}$. Mais recentemente, iniciou-se uma discussão sobre a eficácia da quimioprofilaxia com uma dose de rifampicina ${ }^{(28-29)}$.

Nesse sentido, a proporção de contatos examinados permite conhecer não somente a cobertura da vigilância realizada pelos profissionais de saúde, mas também entender os motivos que levam à agenda inconclusa que o país ainda enfrenta no controle da doença.

Para além da expansão da cobertura da APS, não se pode descartar a necessidade de capacitações profissionais de forma continuada. Segundo Starfield( ${ }^{(30)}$, a qualidade da atenção dispensada ao usuário está diretamente relacionada à capacitação durante a graduação, ao treinamento em serviço e, por último, à experiência profissional com o problema específico.

Entre as limitações do presente estudo, é importante considerar que as informações utilizadas foram obtidas de dados secundários, que podem apresentar inconsistência na qualidade e quantidade das informações. Esse fato ocorre devido a possíveis subnotificações, erros no preenchimento das fichas de notificações e no lançamento dos dados no sistema de informação. Mas, apesar disso, a escolha por esse tipo de fonte reduz os custos operacionais e não inviabiliza a realização de análises.

\section{CONCLUSÃO}

Apesar do declínio da taxa de detecção de novos casos e o aumento da proporção de contatos examinados, percebe-se que a hanseníase ainda nos dias atuais é um significativo problema de saúde pública. Torna-se necessário enfatizar que o decréscimo da taxa de detecção de novos casos podem ser consequência de vários fatores, entre eles uma lacuna no processo de cuidado em saúde. É de suma importância o fortalecimento de políticas de promoção, prevenção e diagnóstico da hanseníase, impactando na melhoria das condições de vida da população, reiterando o importante papel da saúde coletiva para o controle da endemia.

O aumento da proporção de contatos examinados é uma tendência positiva para o estado, uma vez que avalia a capacidade dos serviços em realizar a vigilância de contatos no ano de diagnóstico dos casos novos. No entanto, esse dado deve ser analisado com cautela, uma vez que o percentual apresentado no período está abaixo do esperado pelo Ministério da Saúde. Por fim, este estudo reforça a importância da realização de novos estudos como caminho para subsidiar o desenvolvimento de outras estratégias para o controle da hanseníase.

\section{REFERÊNCIAS}

1. Brasil. Ministério da Saúde. Secretaria de Vigilância em Saúde. Departamento de Vigilância das Doenças Transmissiveis. Guia prático sobre a hanseníase. Brasilia: Ministério da Saúde, 2017. 68 p. [acesso em 25 jul. 2020]. Disponivel em: https:/portalarquivos2.saude.gov.br/images/ pdf/2017/novembro/22/Guia-Pratico-de-Hanseniase-WEB.pdf.2. World health organization. Global leprosy update, 2018: moving towards a leprosy- free world. Weekly Epidemiological Record. Geneva. 2019; 94(35-36):389-412.

2. World health organization. Global leprosy update, 2018: moving towards a leprosy- free world. Weekly Epidemiological Record. Geneva. 2019; 94(35-36):389-412.

3. Brasil. Ministério da Saúde. Secretaria de Vigilância em Saúde. Hanseníase. Boletim epidemiológico, 2018. [acesso em 12 ago. 2020]. Disponível em: https:/www.saude.gov.br/images/pdf/2018/janeiro/31/
2018-004-Hanseniase-publicacao.pdf.

4. Pereira KC, Bueno IC, Lana FCF. Tendência epidemiológica da hanseníase em Minas Gerais (1995-2015). Cogitare enferm. [internet]. 2020; 24:e66109. doi: http:/dx.doi.org/10.5380/ce.v24i0.66109.

5. Rodrigues RN, Leano HAM, Bueno IC, Araújo KMFA, Lana FCF. Áreas de alto risco de hanseníase no Brasil, período 2001-2015. Rev. Bras. Enferm. [Internet]. 2020; 73(3): e20180583. doi: https:/doi.org/10.1590/00347167-2018-0583.

6. Alter A, Grant A, Abel L, Alcais A, Schurr E. Leprosy as a genetic disease. Mammalian Genome, New York. 2011; 22(2):19-31. doi: https:/doi. org/10.1007/s00335-010-9287-1.

7. Masaki T, McGlinchey A, Cholewa-Waclaw J, Qu J, Tomlinson SR, Rambukkana $A$. Innate immune response precedes Mycobacterium leprae- 


\section{REFERÊNCIAS}

-induced reprogramming of adult Schwann cells. Cell Reprogram. 2014; 16(1):9-17. doi: https:/doi.org/10.1089/cell.2013.0064.

8. Sousa GS, Silva RLF, Xavier MB. Hanseníase e Atenção Primária à Saúde: uma avaliação de estrutura do programa. Saúde debate [Internet]. 2017; 41(112):230-242. doi: https:/doi.org/10.1590/01031104201711219.

9. Garbin CAS, Garbin All, Carloni MEOG, Rovida TAS, Martins RJ. The stigma and prejudice of leprosy: influence on the human condition. Rev. Soc. Bras. Med. Trop. [Internet]. 2015; 48(2):194-201. doi: https:/doi. org/0037-8682-0004-2015.

10. Brasil. Ministério da Saúde. Secretaria de Vigilância em Saúde. Departamento de Vigilância das Doenças Transmissiveis. Diretrizes para vigilância, atenção e eliminação da Hanseníase como problema de saúde pública: manual técnico- operacional. Brasilia: Ministério da Saúde. 2016. 58p. [acesso em 20 jun. 2020]. Disponível em: http:/www.saude.gov.br/ images/pdf/2016/fevereiro/04/diretrizes-eliminacao-hanseniase-4fev16-web.pdf.

11. Bratschi MW, Steinmann P, Wickenden A, Gillis TP. Current knowledge on Mycobacterium leprae transmission: a systematic literature review. Lepr Rev. 2015;86(2):142-155.

12. Brasil. Ministério da Saúde. Secretaria de Vigilância em Saúde. Departamento de Análise de Situação de Saúde. Saúde Brasil 2009: uma análise da situação de saúde e da agenda nacional e internacional de prioridades em saúde. Brasilia: Ministério da Saúde, 2010. 368 p. [acesso em 20 jun. 2020]. Disponivel em: http:/svs.aids.gov.br/dantps/centrais-de-conteudos/publicacoes/saude-brasil/saude-brasil-2009-uma-analise-da-situacao-de-saude-e-da-agenda-nacional-e-internacional-de-prioridades-em-saude.pdf.

13. Penna MLF, Grossi MAF, Rocha MCN, Penna GO. Comportamento epidemiológico da hanseníase no Brasil. In: Brasil. Ministério da Saúde. Secretaria de Vigilância em Saúde. Saúde Brasil 2009: uma análise da situação de saúde e da agenda nacional e internacional de prioridades em saúde [Internet]. Brasilia: Ministério da Saúde; 2010. 368p. [acesso em 14 ago. 2020]. Disponivel em: http:/bvsms.saude.gov.br/bvs/publicacoes/saude_brasil_2009.pdf.

14. França Jl, Monteiro CA. Estudo da tendência secular de indicadores de saúde como estratégia de investigação epidemiológica. Rev. Saúde Pública [Internet]. 2000; 34(6 Suppl):5-7. doi: https:/doi.org/10.1590/ S0034-89102000000700002.

15. Instituto Brasileiro de Geografia e Estatística (IBGE). População estimada, 2019. [acesso em 29 abr. 2020]. Disponivel em: https:/www.ibge. gov.br/cidades-e-estados/mg.html.

16. Brasil. Ministério da Saúde. DATASUS. Indicadores de morbidade. Brasilia, 2016. [acesso em 15 mai. 2020]. Disponível em: http:/tabnet. datasus.gov.br/cgi/tabcgi.exe?sinannet/hanseniase/cnv/hanswuf.def.

17. Antunes JLF, Cardoso MRA. Uso da análise de séries temporais em estudos epidemiológicos. Epidemiol. Serv. Saúde [Internet]. 2015; 24(3):565-576. doi: https:/doi.org/10.5123/S167949742015000300024.

18. Brasil. Ministério da Saúde. Conselho Nacional de Saúde. Comissão Nacional de Ética em Pesquisa. Resolução n 466, de 12 de dezembro de
2012. Brasilia: Ministério da Saúde, 2012. [acesso em 20 jun. 2020]. Disponivel em: doi: https:/bvsms.saude.gov.br/bvs/saudelegis/cns/2013/ res0466_12_12_2012.html.

19. Anchieta JJS et al. Análise da tendência dos indicadores da hanseníase em estado brasileiro hiperendêmico, 2001-2015. Rev. Saúde Pública [Internet]. 2019; 53:61. doi: https:/doi.org/10.11606/s15188787.2019053000752.

20. Gomes FBF, Lana FCF, Oliveira RC, Rodrigues RN. Indicadores da hanseníase no Estado de Minas Gerais e sua relação com o índice de desenvolvimento humano municipal e a cobertura da Estratégia da Saúde da Familia. Rev. min. Enferm. 2017; 21:e1063. doi: http:/www.dx.doi. org/10.5935/1415-2762.20170073.

21. Penna MLF, Grossi MAF, Penna GO. Country profile: leprosy in Brazil. Leprosy Review. London. 2013; 84(4):308-315.

22. Nery JS et al. Efeito dos Programas Brasileiros de Transferência Condicional de Renda e Atenção Primária à Saúde na Taxa de Detecção de Novos Casos de Hanseníase. PLoS NegI Trop Dis. 2014; 8(11):e3357. doi: https:/doi.org/10.1371/journal.pntd.0003357.

23. Souza CDF, Matos TS. Análise de tendência dos indicadores de monitoramento e avaliação da qualidade dos serviços de hanseníase em município prioritário do Nordeste brasileiro. Rev Bras Pesqui Saúde. 2017;19(4):75-83. doi: 10.21722/rbps.v19i4.19806.

24. Abeje T, Negera E, Kebede E, Hailu T, Hassen I, Lema T, et al. Performance of general health workers in leprosy control activities at public health facilities in Amhara and Oromia States, Ethiopia. BMC Health Serv Res. 2016; 16:122. doi: https:/doi.org/10.1186/s12913-016-1329-2.

25. Siddiqui MR et al. Integration of leprosy elimination into primary health care in Orissa, India. PLoS One. 2009; 4(12): e8351. doi: https:/doi. org/10.1371/journal.pone.0008351.

26. Romanholo HSB et al. Vigilância de contatos intradomiciliares de hanseníase: perspectiva do usuário em município hiperendêmico. Rev. Bras. Enf. 2018; 71(1):175-81. http:/dx.doi.org/10.1590/0034-71672016-0607.

27. Gillini L, Cooreman E, Wood T, Pemmaraju VR, Saunderson P. Global practices in regard to implementation of preventive measures for leprosy. PLoS Neglected Tropical Diseases. San Francisco. 2017; 11(5):e0005399.

https:/doi.org/10.1371/journal.pntd.0005399.

28. Lockwood DNJ, Krishnamurthy P, Kumar B, Penna G. Single-dose rifampicin chemoprophylaxis protects those who need it least and is not a cost-effective intervention. PLoS Neglected Tropical Diseases. San Francisco. 2018; 12(6):e0006403. https:/doi.org/10.1371/journal. pntd.0006403.

29. Tiwari A, Dandel S, Djupuri R, Mieras L, Richardus JH. Population-wide administration of single dose rifampicin for leprosy prevention in isolated communities: a three year follow-up feasibility study in Indonesia. BMC Infectious Diseases. London. 2018; 18(1):324.

30. Starfield B. Atenção primária: equilibrio entre necessidades de saúde, serviços e tecnologia. Brasilia: UNESCO, Ministério da Saúde, 2002. 726p. [acesso em 12 ago. 2020]. Disponivel em: https:/www.nescon.medicina. ufmg.br/biblioteca/imagem/0253.pdf. 